CALT-68-2566

UCSD/PTH-05-11

June 2005

\title{
Minimal Flavor Violation in the Lepton Sector
}

\author{
Vincenzo Cirigliano $^{a}$, Benjamín Grinstein ${ }^{b}$, Gino Isidori $^{c}$, Mark B. Wise ${ }^{a}$ \\ ${ }^{a}$ California Institute of Technology, Pasadena, CA 91125 \\ ${ }^{b}$ Department of Physics, University of California at San Diego, La Jolla, CA 92093 \\ ${ }^{c}$ INFN, Laboratori Nazionali di Frascati, Via E. Fermi 40, I-00044 Frascati, Italy
}

\begin{abstract}
We extend the notion of Minimal Flavor Violation to the lepton sector. We introduce a symmetry principle which allows us to express lepton flavor violation in the charged lepton sector in terms of neutrino masses and mixing angles. We explore the dependence of the rates for flavor changing radiative charged lepton decays $\left(\ell_{i} \rightarrow \ell_{j} \gamma\right)$ and $\mu$-to-e conversion in nuclei on the scales for total lepton number violation, lepton flavor violation and the neutrino masses and mixing angles. Measurable rates are obtained when the scale for total lepton number violation is much larger than the scale for lepton flavor violation.
\end{abstract}

\section{Introduction}

With the discovery of neutrino masses and mixing it has been clearly established that lepton flavor is not conserved. The smallness of neutrino masses also provides a strong indication in favor of the non-conservation of total lepton number, although this information cannot be directly extracted from data yet. In analogy to what happens in the quark sector, the non-conservation of lepton flavor points toward the existence of lepton flavor violating (LFV) processes with charged leptons, which however have not been observed so far.

Within the Standard Model (SM), the flavor violation in the quark sector is induced by Yukawa interactions. These do not break the baryon number and, to a good approximation, leave the neutral currents flavor diagonal. This fact puts very stringent constraints on the structure of possible new degrees of freedom. New, flavor-dependent interactions beyond the SM can readily be ruled out if they contribute significantly to flavor changing neutral currents (FCNC). One way to evade this constraint is to assume that all the new degrees of freedom, or at least all the new particles carrying flavor 
quantum numbers, are very heavy. This solution is theoretically unappealing and largely non-testable. A more appealing scenario is to assume that the new flavor-changing couplings appearing in SM extensions are suppressed by some symmetry principle. The most restrictive and predictive symmetry principle of this type is the so-called Minimal Flavor Violation (MFV) hypothesis [1, 2, 3]: the assumption that the SM Yukawa couplings are the only sources of quark-flavor symmetry breaking. In this case all the cancellations that render FCNC automatically small in the SM apply just as well to the new degrees of freedom, allowing for very reasonable mass scales of the new particles. Interestingly, the MFV hypothesis can be formulated in a very general way in terms of an effective field theory [3], without the need of specifying the nature of the new degrees of freedom. In a theory where the new degrees of freedom also carry lepton flavor quantum numbers, it is natural to expect that a similar mechanism occurs also in the lepton sector.

In this paper we extend the notion of MFV to the lepton sector. In other words, we define and analyze a consistent class of SM extensions where the sources of LFV are linked in a minimal way to the known structure of the neutrino and charged-lepton mass matrices. This allows us to address in a general way several interesting questions. In particular, we shall analyze the general requirements about the scale of new physics under which we can expect observable effects in low-energy rare LFV processes, such as $\mu \rightarrow e \gamma$ and $\mu$-to-e conversion in nuclei, without requiring the existence of new uncontrollable sources of lepton flavor mixing. We shall also identify some model-independent relations among different LFV observables which could allow to falsify this general hypothesis about the flavor structure of physics beyond the SM.

The large difference between charged lepton and neutrino masses is naturally attributed to the breaking of total lepton number. This assumption has very important consequences to estimate the overall size of the LFV terms. As we shall show, only by decoupling the mechanisms of lepton flavor mixing and lepton number violation can we generate sizable LFV amplitudes in the charged-lepton sector. Since lepton flavor and lepton number correspond to two independent symmetry groups, this decoupling can naturally be implemented in an effective field theory approach with the minimal particle content, namely without introducing right-handed neutrino fields. However, in most explicit SM extensions this result is achieved by means of the see-saw mechanism with heavy right-handed neutrinos. For this reason, we shall consider two main possibilities in order to define the minimal sources of flavor symmetry breaking in the lepton sector: i) a scenario without right-handed neutrinos, where the (left-handed) Majorana mass matrix is the only irreducible source of flavor symmetry breaking; ii) a scenario with right-handed neutrinos, where the Yukawa couplings define the irreducible sources of flavor symmetry breaking and the (right-handed) Majorana mass matrix has a trivial flavor structure.

\section{Minimal breaking of the lepton flavor symmetry}

In the absence of Yukawa couplings, the flavor symmetry of the quark sector of the SM would be $S U(3)_{Q} \times S U(3)_{U} \times S U(3)_{D}$ corresponding to individual rotations of the $Q_{L}^{i}$, $u_{R}^{i}$ and $d_{R}^{i}$ fields (the left-handed quark doublet and the two right-handed quark singlets) 
for $i=1,2,3$. Models with MFV have only two independent sources of breaking of this group, namely the two Yukawa couplings $\lambda_{U}$ and $\lambda_{D}$. Each of them breaks the symmetry in a specific way: in the spurion sense, $\lambda_{U}$ transforms as a $(3, \overline{3}, 1)$ while $\lambda_{D}$ as a $(3,1, \overline{3})$. In MFV models any higher dimension operator that describes long distance remnants of very short distance physics must be invariant under the full flavor symmetry group when the couplings $\lambda_{U}$ and $\lambda_{D}$ are taken to transform as spurions as above 3].

In order to define a similar minimal flavor violating structure for the leptons, we first need to specify the field content of the theory in the lepton sector. As anticipated, we shall consider two cases:

1. Minimal field content: three left-handed lepton doublets $L_{L}^{i}$ and three right-handed charged lepton singlets $e_{R}^{i}$ (SM field content). In this case the lepton flavor symmetry group is

$$
G_{\mathrm{LF}}=S U(3)_{L} \times S U(3)_{E}
$$

The lepton sector is also invariant under two $U(1)$ symmetries, which can be identified with total lepton number, $U(1)_{\mathrm{LN}}$, and the weak hypercharge.

2. Extended field content: three right-handed neutrinos, $\nu_{R}^{i}$, in addition to the SM fields. In this case the field content of the lepton sector is very similar to that of the quark sector, with a maximal flavor group $G_{\mathrm{LF}} \times S U(3)_{\nu_{R}}$.

In the following we shall define separately the assumptions of Minimal Lepton Flavor Violation (MLFV) in these two cases.

\subsection{Minimal Field Content}

In this case the minimal choice for the neutrino mass matrix is a left-handed Majorana mass term transforming as $(6,1)$ under $G_{\mathrm{LF}}$. Because of the $S U(2)_{L}$ gauge symmetry, this mass term cannot be generated by renormalizable interactions. Moreover, the absence of right-handed neutrino fields requires the breaking of total lepton number. We define the MLFV hypothesis in this case as follows:

1. The breaking of the $U(1)_{\mathrm{LN}}$ is independent from the breaking of the lepton flavor symmetry $\left(G_{\mathrm{LF}}\right)$ and is associated to a very high scale $\Lambda_{\mathrm{LN}}$.

2. There are only two irreducible sources of lepton-flavor symmetry breaking, $\lambda_{e}^{i j}$ and $g_{\nu}^{i j}$, defined by ${ }^{1}$

$$
\begin{aligned}
\mathcal{L}_{\text {Sym.Br. }} & =-\lambda_{e}^{i j} \bar{e}_{R}^{i}\left(H^{\dagger} L_{L}^{j}\right)-\frac{1}{2 \Lambda_{\mathrm{LN}}} g_{\nu}^{i j}\left(\bar{L}_{L}^{c i} \tau_{2} H\right)\left(H^{T} \tau_{2} L_{L}^{j}\right)+\text { h.c. } \\
& \rightarrow-v \lambda_{e}^{i j} \bar{e}_{R}^{i} e_{L}^{j}-\frac{v^{2}}{2 \Lambda_{\mathrm{LN}}} g_{\nu}^{i j} \bar{\nu}_{L}^{c i} \nu_{L}^{j}+\text { h.c. }
\end{aligned}
$$

The smallness of the neutrino mass is attributed to the smallness of $v / \Lambda_{\mathrm{LN}}$, while $g_{\nu}^{i j}$ can have entries of $\mathcal{O}(1)$ as in the standard see-saw mechanism.

\footnotetext{
${ }^{1}$ Throughout this paper we use four-component spinor fields, and $\psi^{c}=-i \gamma^{2} \psi^{*}$ denotes the charge conjugate of the field $\psi$. We also use $v=\left\langle H^{0}\right\rangle \simeq 174 \mathrm{GeV}$.
} 
The transformation properties of the lepton field under $G_{\mathrm{LF}}$ are

$$
L_{L} \rightarrow V_{L} L_{L}, \quad e_{R} \rightarrow V_{R} e_{R} .
$$

Thus the Lagrangian (21) is formally invariant under this symmetry if the matrices $\lambda_{e}^{i j}$ and $g_{\nu}^{i j}$ are taken as spurions transforming as

$$
\lambda_{e} \rightarrow V_{R} \lambda_{e} V_{L}^{\dagger}, \quad g_{\nu} \rightarrow V_{L}^{*} g_{\nu} V_{L}^{\dagger} .
$$

Since we are interested in LFV processes with external charged leptons, we can use the $G_{\mathrm{LF}}$ invariance and rotate the fields in the basis where $\lambda_{e}$ is flavor diagonal. In such basis

$$
\begin{aligned}
& \lambda_{e}=\frac{m_{\ell}}{v}=\frac{1}{v} \operatorname{diag}\left(m_{e}, m_{\mu}, m_{\tau}\right), \\
& g_{\nu}=\frac{\Lambda_{\mathrm{LN}}}{v^{2}} \hat{U}^{*} m_{\nu} \hat{U}^{\dagger}=\frac{\Lambda_{\mathrm{LN}}}{v^{2}} \hat{U}^{*} \operatorname{diag}\left(m_{\nu_{1}}, m_{\nu_{2}}, m_{\nu_{3}}\right) \hat{U}^{\dagger},
\end{aligned}
$$

where $\hat{U}$ is the Pontecorvo-Maki-Nakagawa-Sakata (PMNS) mixing matrix. The latter can be written as $\hat{U}=U_{e_{L}}^{\dagger} U_{\nu_{L}}$ in terms of the unitary matrices which connect a generic basis of the lepton fields to the mass-eigenstate basis (denoted by a prime):

$$
e_{L}=U_{e_{L}} e_{L}^{\prime}, \quad e_{R}=U_{e_{R}} e_{R}^{\prime}, \quad \nu_{L}=U_{\nu_{L}} \nu_{L}^{\prime} .
$$

In the basis defined by (6) the simplest spurion combination transforming as $(8,1)$ under $G_{\mathrm{LF}}$, or the coupling which controls the amount of LFV in the charged-lepton sector, is ${ }^{2}$

$$
\left.\Delta\right|_{\text {minimal }}=g_{\nu}^{\dagger} g_{\nu}=\frac{\Lambda_{\mathrm{LN}}^{2}}{v^{4}} \hat{U} m_{\nu}^{2} \hat{U}^{\dagger} .
$$

\section{2 $\quad$ Extended Field Content}

The second scenario we consider has three right-handed neutrinos in addition to the SM fields, with a maximal flavor group $G_{\mathrm{LF}} \times S U(3)_{\nu_{R}}$. There is a large freedom in deciding how to break this group in order to generate the observed masses and mixing. In addition to the standard Yukawa coupling for the charged leptons, in principle we can introduce neutrino mass terms transforming as $(6,1,1),(1,1,6)$, and $(\overline{3}, 1,3)$. Since we are interested in a minimal scenario, with unambiguous links between the irreducible sources of flavor-symmetry breaking and the observable couplings in the neutrino mass matrix, we must choose only one of these possibilities. In order to distinguish this scenario from the previous one, and guided by the structure of explicit models with see-saw mechanism (see e.g. Ref. [4), we make the following assumptions:

1. The right-handed neutrino mass term breaks $S U(3)_{\nu_{R}}$ to $O(3)_{\nu_{R}}$, namely is proportional to the identity matrix in flavor space:

$$
\mathcal{L}_{\nu_{R} \text {-mass }}=-\frac{1}{2} M_{\nu}^{i j} \bar{\nu}_{R}^{c i} \nu_{R}^{j}+\text { h.c. } \quad \text { with } \quad M_{\nu}^{i j}=M_{\nu} \delta^{i j} .
$$

\footnotetext{
${ }^{2} g_{\nu}^{\dagger} g_{\nu}$ also contains a $(1,1)$ piece under $G_{\mathrm{LF}}$. However, it does not contribute to lepton flavor violation. Note also that if $\mathrm{CP}$ were an exact symmetry, $V_{L}$ in Eq. (5) would be required to be real, and therefore $\Delta_{\text {minimal }}=g_{\nu}$.
} 
2. The right-handed neutrino mass is the only source of $U(1)_{\mathrm{LN}}$ breaking and its scale is large compared to the electroweak symmetry breaking scale: $\left|M_{\nu}\right| \gg v$.

3. The remaining lepton-flavor symmetry is broken only by two irreducible sources, $\lambda_{e}^{i j}$ and $\lambda_{\nu}^{i j}$, defined by

$$
\begin{aligned}
\mathcal{L}_{\text {Sym.Br. }} & =-\lambda_{e}^{i j} \bar{e}_{R}^{i}\left(H^{\dagger} L_{L}^{j}\right)+i \lambda_{\nu}^{i j} \bar{\nu}_{R}^{i}\left(H^{T} \tau_{2} L_{L}^{j}\right)+\text { h.c. } \\
& \rightarrow-v \lambda_{e}^{i j} \bar{e}_{R}^{i} e_{L}^{j}-v \lambda_{\nu}^{i j} \bar{\nu}_{R}^{i} \nu_{L}^{j}+\text { h.c. }
\end{aligned}
$$

The theory is thus formally invariant under the group $G_{\mathrm{LF}} \times O(3)_{\nu_{R}}$, with the following transformation properties for fields and spurions:

$$
\begin{gathered}
L_{L} \rightarrow V_{L} L_{L}, \quad e_{R} \rightarrow V_{R} e_{R}, \quad \nu_{R} \rightarrow O_{\nu} \nu_{R}, \\
\lambda_{e} \rightarrow V_{R} \lambda_{e} V_{L}^{\dagger}, \quad \lambda_{\nu} \rightarrow O_{\nu} \lambda_{\nu} V_{L}^{\dagger} .
\end{gathered}
$$

At low energies the heavy right-handed neutrinos are integrated out, generating an effective left-handed Majorana mass matrix,

$$
m_{\nu}=\frac{v^{2}}{M_{\nu}} \lambda_{\nu}^{T} \lambda_{\nu}
$$

as in the minimal scenario. Once we identify $M_{\nu}$ with $\Lambda_{\mathrm{LN}}$, the two scenarios are perfectly equivalent as far as field content and operator structure are concerned. However, in the extended case the effective left-handed neutrino mass matrix is not an irreducible source of flavor breaking, since it can be expressed in terms of the neutrino Yukawa coupling, $\lambda_{\nu}$ :

$$
\frac{v^{2} g_{\nu}}{\Lambda_{\mathrm{LN}}} \quad[\text { minimal case }] \leftrightarrow \frac{v^{2}}{M_{\nu}} \lambda_{\nu}^{T} \lambda_{\nu} \quad \text { [extended case] }
$$

This has important consequences for LFV processes: the simplest spurion combination transforming as $(8,1)$ under $G_{\mathrm{LF}}$ is now $\lambda_{\nu}^{\dagger} \lambda_{\nu}$ and is not quadratic in the neutrino masses as $g_{\nu}^{\dagger} g_{\nu}$ in Eq. (8). Since $\lambda_{\nu}^{\dagger} \lambda_{\nu}$ and $\lambda_{\nu}^{T} \lambda_{\nu}$ are not necessarily diagonalized by the same orthogonal transformation, this also implies that the connection between LFV processes and the observables in the neutrino sector is not completely unambiguous in this case. We can overcome this difficulty by neglecting $\mathrm{CP}$ violation in the neutrino mass matrix. With this additional assumption, $\lambda_{\nu}^{\dagger} \lambda_{\nu}$ and $\lambda_{\nu}^{T} \lambda_{\nu}$ are the same (real) combination diagonalized by the (real) PMNS matrix:

$$
\left.\Delta\right|_{\text {extended }}=\lambda_{\nu}^{\dagger} \lambda_{\nu} \stackrel{\mathrm{CP} \text { limit }}{\longrightarrow} \frac{M_{\nu}}{v^{2}} \hat{U} m_{\nu} \hat{U}^{\dagger} .
$$

\section{Operator analysis for LFV processes}

In addition to the SM leptons, we assume that at some scale $\Lambda_{\mathrm{LFV}}$ above the electroweak scale and well below $\Lambda_{\mathrm{LN}}$ (or $M_{\nu}$ ) there are new degrees of freedom carrying lepton flavor quantum numbers. Integrating them out, their effect will show up at low energies as a series of non-renormalizable operators suppressed by inverse powers of $\Lambda_{\mathrm{LFV}}$. According 
to the MLFV hypothesis, these operators must be constructed in terms of SM fields and the spurions $\lambda_{e}$ and $g_{\nu}$ (or $\lambda_{\nu}$ ), and must be invariant under $G_{\mathrm{LF}}$ when the spurions transform as in Eqs. (15) or (11).

We are interested in those operators of dimension five and six that could lead to LFV process with charged leptons. These operators must conserve total lepton number, otherwise they would be suppressed by the large $U(1)_{\text {LN }}$ breaking scale. As a consequence, no dimension-five term turns out to be relevant. For processes involving only two lepton fields, such as $\mu \rightarrow e \gamma$ and $\mu$-to-e conversion, the basic building blocks are the bilinears $\bar{L}_{L}^{i} \Gamma L_{L}^{j}, \bar{e}_{R}^{i} \Gamma L_{L}^{j}$ and $\bar{e}_{R}^{i} \Gamma e_{R}^{j}$. Their indexes must be contracted with spurion combinations transforming under $G_{\mathrm{LF}}$ as $(8,1),(\overline{3}, 3)$ and $(1,8)$, respectively. Combinations of this type are

$$
\begin{aligned}
& \Delta, \lambda_{e}^{\dagger} \lambda_{e}, \Delta^{2}, \lambda_{e}^{\dagger} \lambda_{e} \Delta, \ldots \\
& \lambda_{e}, \lambda_{e} \Delta, \lambda_{e} \lambda_{e}^{\dagger} \lambda_{e}, \ldots \\
& \lambda_{e} \lambda_{e}^{\dagger}, \lambda_{e} \Delta \lambda_{e}^{\dagger}, \ldots
\end{aligned}
$$

where $\Delta$ is defined in Eqs. (8) or (14) for the two scenarios. Given the smallness of $\lambda_{e}$ (which is unambiguously fixed by charged lepton masses), we can safely neglect terms which are of second order in $\lambda_{e}$. We shall also assume that the entries of $\Delta$ are perturbative, retaining only linear terms in this effective coupling. In this limit the only relevant LFV couplings are $\Delta$ and $\lambda_{e} \Delta$. Moreover, we work only to linear order in the quark Yukawa couplings, $\lambda_{U}$ and $\lambda_{D}$.

The resulting dimension-six operators bilinear in the lepton fields can be written as

$$
\begin{array}{rlrl}
O_{L L}^{(1)} & =\bar{L}_{L} \gamma^{\mu} \Delta L_{L} H^{\dagger} i D_{\mu} H & & O_{R L}^{(1)}=g^{\prime} H^{\dagger} \bar{e}_{R} \sigma^{\mu \nu} \lambda_{e} \Delta L_{L} B_{\mu \nu} \\
O_{L L}^{(2)} & =\bar{L}_{L} \gamma^{\mu} \tau^{a} \Delta L_{L} H^{\dagger} \tau^{a} i D_{\mu} H & & O_{R L}^{(2)}=g H^{\dagger} \bar{e}_{R} \sigma^{\mu \nu} \tau^{a} \lambda_{e} \Delta L_{L} W_{\mu \nu}^{a} \\
O_{L L}^{(3)} & =\bar{L}_{L} \gamma^{\mu} \Delta L_{L} \bar{Q}_{L} \gamma_{\mu} Q_{L} & & O_{R L}^{(3)}=\left(D_{\mu} H\right)^{\dagger} \bar{e}_{R} \lambda_{e} \Delta D_{\mu} L_{L} \\
O_{L L}^{(4 d)} & =\bar{L}_{L} \gamma^{\mu} \Delta L_{L} \bar{d}_{R} \gamma_{\mu} d_{R} & & O_{R L}^{(4)}=\bar{e}_{R} \lambda_{e} \Delta L_{L} \bar{Q}_{L} \lambda_{D} d_{R} \\
O_{L L}^{(4 u)} & =\bar{L}_{L} \gamma^{\mu} \Delta L_{L} \bar{u}_{R} \gamma_{\mu} u_{R} & & O_{R L}^{(5)}=\bar{e}_{R} \sigma^{\mu \nu} \lambda_{e} \Delta L_{L} \bar{Q}_{L} \sigma_{\mu \nu} \lambda_{D} d_{R} \\
O_{L L}^{(5)} & =\bar{L}_{L} \gamma^{\mu} \tau^{a} \Delta L_{L} \bar{Q}_{L} \gamma_{\mu} \tau^{a} Q_{L} & & O_{R L}^{(6)}=\bar{e}_{R} \lambda_{e} \Delta L_{L} \bar{u}_{R} \lambda_{U}^{\dagger} i \tau^{2} Q_{L} \\
& & O_{R L}^{(7)}=\bar{e}_{R} \sigma^{\mu \nu} \lambda_{e} \Delta L_{L} \bar{u}_{R} \sigma_{\mu \nu} \lambda_{U}^{\dagger} i \tau^{2} Q_{L}
\end{array}
$$

We have omitted operators of the type $H^{\dagger} \bar{e}_{R} \lambda_{e} L_{L} H^{\dagger} H$, which correct the charged lepton mass matrix but produce no FCNC interactions.

The operator $O_{R L}^{(3)}$ does not contribute to the radiative lepton flavor changing decays $\ell_{i} \rightarrow \ell_{j} \gamma$, and its contribution to $\mu$-e conversion is suppressed by $m_{e} m_{\mu} / v^{2}$. The MFV assumption in the quark sector requires the RL operators with a quark current to contain at least one power of the quark Yukawa couplings $\lambda_{D}$ or $\lambda_{U}$. Only the top-quark Yukawa is non-negligible, and hence, for the low energy processes we consider $O_{R L}^{(4)}-O_{R L}^{(7)}$ can be neglected.

Since the top quark Yukawa is order one, in principle, operators involving higher orders in $\lambda_{U}$ could be important. They induce non-negligible FCNC currents in the down-quark sector of the type $V_{\mathrm{CKM} t i} V_{\mathrm{CKM}_{t j}}^{*} \bar{d}_{L}^{i} \gamma^{\mu} d_{L}^{j}$ [3]. For $\mu$-to-e conversion only the 
coupling to light quarks is relevant, and this additional contribution is suppressed by $\left|V_{\mathrm{CKM} t d}\right|^{2} \ll 1$.

In this paper we shall analyze the phenomenological consequences of the MLFV hypothesis only in processes involving two lepton fields, for which significant prospects of experimental improvements are foreseen in the near future [5, 6]. However, one can in principle apply it also to four-lepton processes, such as $\mu \rightarrow 3 e$. In this case one needs to extend the operator basis (18) including the generalization of $O_{L L}^{(3-5)}$, namely

$$
\bar{L}_{L} \gamma^{\mu} \Delta L_{L} \bar{L}_{L} \gamma_{\mu} L_{L}, \quad \bar{L}_{L} \gamma^{\mu} \Delta L_{L} \bar{e}_{R} \gamma_{\mu} e_{R}, \quad \bar{L}_{L} \gamma^{\mu} \tau^{a} \Delta L_{L} \bar{L}_{L} \gamma_{\mu} \tau^{a} L_{L}
$$

and also new structures of the type

$$
\bar{L}_{L}^{c} g_{\nu} L_{L} \bar{L}_{L} g_{\nu}^{\dagger} L_{L}^{c} \quad \text { or } \quad \bar{L}_{L}^{c} \lambda_{\nu}^{T} \lambda_{\nu} L_{L} \bar{L}_{L} \lambda_{\nu}^{\dagger} \lambda_{\nu}^{*} L_{L}^{c}
$$

\subsection{Explicit structure of the LFV couplings}

Given the structure of operators in Eq. (18), it is clear that the strength of LFV processes is determined by the entries of the matrix $\Delta$ in the mass-eigenstate basis of charged leptons. These are listed below for the two scenarios we are considering, and for the two allowed structures (normal and inverted hierarchy) of the neutrino mass matrix:

1. Minimal field content. According to Eq. (86), we have

$$
\Delta_{i j}=\frac{\Lambda_{\mathrm{LN}}^{2}}{v^{4}}\left[m_{\nu_{1}}^{2} \delta_{i j}+\hat{U}_{i 2} \hat{U}_{j 2}^{*} \Delta m_{\mathrm{sol}}^{2} \pm \hat{U}_{i 3} \hat{U}_{j 3}^{*} \Delta m_{\mathrm{atm}}^{2}\right]
$$

where $\Delta m_{\mathrm{atm}}^{2}$ and $\Delta m_{\text {sol }}^{2}$ denote the squared mass differences deduced from atmospheric and solar neutrino data, respectively. The plus sign corresponds to normal hierarchy $\left(m_{\nu_{1}}<m_{\nu_{2}} \ll m_{\nu_{3}}\right)$, while the minus one to the inverted case $\left(m_{\nu_{3}} \ll m_{\nu_{1}}<m_{\nu_{2}}\right)$. Explicitly, using the PDG notation of the PMNS matrix (we adopt the convention that $s_{13} \geq 0$ and $0 \leq \delta<2 \pi$ ) [9], we find

$$
\begin{aligned}
\Delta_{\mu e} & =\frac{\Lambda_{\mathrm{LN}}^{2}}{v^{4}} \frac{1}{\sqrt{2}}\left(s c \Delta m_{\mathrm{sol}}^{2} \pm s_{13} e^{i \delta} \Delta m_{\mathrm{atm}}^{2}\right) \equiv \frac{\Lambda_{\mathrm{LN}}^{2}}{v^{2}} a_{\mu e}, \\
\Delta_{\tau e} & =\frac{\Lambda_{\mathrm{LN}}^{2}}{v^{4}} \frac{1}{\sqrt{2}}\left(-s c \Delta m_{\mathrm{sol}}^{2} \pm s_{13} e^{i \delta} \Delta m_{\mathrm{atm}}^{2}\right) \equiv \frac{\Lambda_{\mathrm{LN}}^{2}}{v^{2}} a_{\tau e} \\
\Delta_{\tau \mu} & =\frac{\Lambda_{\mathrm{LN}}^{2}}{v^{4}} \frac{1}{2}\left(-c^{2} \Delta m_{\mathrm{sol}}^{2} \pm \Delta m_{\mathrm{atm}}^{2}\right) \equiv \frac{\Lambda_{\mathrm{LN}}^{2}}{v^{2}} a_{\tau \mu},
\end{aligned}
$$

where we have assumed maximal mixing for the atmospheric case and $s$ and $c$ denote sine and cosine of the solar mixing angle. In a given scenario for the spectrum (normal or inverted), the dimensionless couplings $a_{i j}$ are completely fixed by oscillation experiments modulo the dependence on the combination $s_{13} e^{i \delta}$.

2. Extended field content. According to Eq. (14), assuming CP conservation in the lepton sector we have

$$
\Delta_{i j}=\frac{M_{\nu}}{v^{2}}\left[m_{\nu_{1}} \delta_{i j}+\hat{U}_{i 2} \hat{U}_{j 2}\left(m_{\nu_{2}}-m_{\nu_{1}}\right)+\hat{U}_{i 3} \hat{U}_{j 3}\left(m_{\nu_{3}}-m_{\nu_{1}}\right)\right] .
$$


Note that the assumption of CP conservation forces us to choose the PMNS phase $\delta=0$ or $\pi$. Hence,

$$
\begin{aligned}
\Delta_{\mu e} & =\frac{M_{\nu}}{v^{2}} \frac{1}{\sqrt{2}}\left[\operatorname{sc}\left(m_{\nu_{2}}-m_{\nu_{1}}\right) \pm s_{13}\left(m_{\nu_{3}}-m_{\nu_{1}}\right)\right] \equiv \frac{M_{\nu}}{v} b_{\mu e} \\
\Delta_{\tau e} & =\frac{M_{\nu}}{v^{2}} \frac{1}{\sqrt{2}}\left[-s c\left(m_{\nu_{2}}-m_{\nu_{1}}\right) \pm s_{13}\left(m_{\nu_{3}}-m_{\nu_{1}}\right)\right] \equiv \frac{M_{\nu}}{v} b_{\tau e} \\
\Delta_{\tau \mu} & =\frac{M_{\nu}}{v^{2}} \frac{1}{2}\left[-c^{2}\left(m_{\nu_{2}}-m_{\nu_{1}}\right)+\left(m_{\nu_{3}}-m_{\nu_{1}}\right)\right] \equiv \frac{M_{\nu}}{v} b_{\tau \mu}
\end{aligned}
$$

where the + and - signs correspond to $\delta=0$ and $\pi$, respectively. In the normal hierarchy case ( $\nu_{1}$ is the lightest neutrino), one has:

$$
m_{\nu_{2}}-m_{\nu_{1}} \stackrel{m_{\nu_{1} \rightarrow 0}}{\longrightarrow} \sqrt{\Delta m_{\mathrm{sol}}^{2}}, \quad m_{\nu_{3}}-m_{\nu_{1}} \stackrel{m_{\nu_{1} \rightarrow 0}}{\longrightarrow} \sqrt{\Delta m_{\mathrm{atm}}^{2}},
$$

while in the inverted hierarchy case ( $\nu_{3}$ is the lightest neutrino)

$$
m_{\nu_{2}}-m_{\nu_{1}} \stackrel{m_{\nu_{3} \rightarrow 0}}{\longrightarrow} \frac{\Delta m_{\mathrm{sol}}^{2}}{2 \sqrt{\Delta m_{\mathrm{atm}}^{2}}}, \quad m_{\nu_{3}}-m_{\nu_{1}} \stackrel{m_{\nu_{3} \rightarrow 0}}{\longrightarrow}-\sqrt{\Delta m_{\mathrm{atm}}^{2}} .
$$

After using input from oscillation experiments, the couplings $b_{i j}$ still depend on the spectrum ordering, the lightest neutrino mass, and the value of $s_{13}$ (the dependence from $\delta$ has disappeared because of the assumption of CP conservation).

\section{Phenomenology}

We are now ready to analyze the phenomenological implications of the new LFV operators. In particular, we are interested in answering the following questions: (i) under which conditions on the new physics scales $\Lambda_{\mathrm{LN}}$ ( or $M_{\nu}$ ) and $\Lambda_{\mathrm{LFV}}$ can we expect observable effects in low energy reactions and therefore positive signals in forthcoming experiments? (ii) is there a specific pattern in the decay rates predicted by MLFV? Can we use it to falsify the assumption of minimal flavor violation?

In order to address these issues, we will study the rates for $\mu \rightarrow e$ conversion in nuclei $\Gamma_{\text {conv }}^{A} \equiv \Gamma\left(\mu^{-}+A(N, Z) \rightarrow e^{-}+A(N, Z)\right)$, experimentally normalized to the capture rate $\Gamma_{\text {capt }}^{A} \equiv \Gamma\left(\mu^{-}+A(Z, N) \rightarrow \nu_{\mu}+A(Z-1, N+1)\right)$, and the radiative decays $\mu \rightarrow e \gamma$, $\tau \rightarrow \mu \gamma, \tau \rightarrow e \gamma$. Throughout, we will use normalized branching fractions defined as:

$$
B_{\mu \rightarrow e}^{A} \equiv \frac{\Gamma_{\text {conv }}^{A}}{\Gamma_{\text {capt }}^{A}}, \quad B_{\ell_{i} \rightarrow \ell_{j} \gamma} \equiv \frac{\Gamma\left(\ell_{i} \rightarrow \ell_{j} \gamma\right)}{\Gamma\left(\ell_{i} \rightarrow \ell_{j} \nu_{i} \bar{\nu}_{j}\right)} .
$$

The starting point of our analysis is the effective Lagrangian generated at a scale $\Lambda_{\mathrm{LFV}}$

$$
\mathcal{L}=\frac{1}{\Lambda_{\mathrm{LFV}}^{2}} \sum_{i=1}^{5} c_{L L}^{(i)} O_{L L}^{(i)}+\frac{1}{\Lambda_{\mathrm{LFV}}^{2}}\left(\sum_{j=1}^{2} c_{R L}^{(j)} O_{R L}^{(j)}+\text { h.c. }\right)
$$

In principle one should evolve this Lagrangian down to the mass of the decaying particles. However, for the purpose of the present work we shall neglect the effect of electroweak 


\begin{tabular}{|c|c|c|c|}
\hline$\Delta m_{\mathrm{sol}}^{2}$ & $\Delta m_{\mathrm{atm}}^{2}$ & $\theta_{\text {sol }}$ & $s_{13}^{\max }$ \\
\hline $8.0 \times 10^{-5} \mathrm{eV}^{2}$ & $2.5 \times 10^{-3} \mathrm{eV}^{2}$ & $33^{\circ}$ & 0.25 \\
\hline
\end{tabular}

Table 1: Reference values of neutrino mixing parameters used in the phenomenological analysis (for a detailed discussion see e.g. [8]).

corrections and treat the $c^{(i)}$ as effective Wilson coefficients renormalized at the low scale. In terms of these we find (in the limit $m_{\ell_{j}} \ll m_{\ell_{i}}$ )

$$
B_{\ell_{i} \rightarrow \ell_{j} \gamma}=384 \pi^{2} e^{2} \frac{v^{4}}{\Lambda_{\mathrm{LFV}}^{4}}\left|\Delta_{i j}\right|^{2}\left|c_{R L}^{(2)}-c_{R L}^{(1)}\right|^{2}
$$

and

$$
\begin{aligned}
B_{\mu \rightarrow e}^{A} & =\frac{32 G_{F}^{2} m_{\mu}^{5}}{\Gamma_{\mathrm{capt}}^{A}} \frac{v^{4}}{\Lambda_{\mathrm{LFV}}^{4}}\left|\Delta_{\mu e}\right|^{2} \mid\left(\left(\frac{1}{4}-s_{w}^{2}\right) V^{(p)}-\frac{1}{4} V^{(n)}\right)\left(c_{L L}^{(1)}+c_{L L}^{(2)}\right) \\
& +\frac{3}{2}\left(V^{(p)}+V^{(n)}\right) c_{L L}^{(3)}+\left(V^{(p)}+\frac{1}{2} V^{(n)}\right) c_{L L}^{(4 u)}+\left(\frac{1}{2} V^{(p)}+V^{(n)}\right) c_{L L}^{(4 d)} \\
& +\frac{1}{2}\left(-V^{(p)}+V^{(n)}\right) c_{L L}^{(5)}-\left.\frac{e D}{4}\left(c_{R L}^{(2)}-c_{R L}^{(1)}\right)^{*}\right|^{2}
\end{aligned}
$$

where we use the notation of Ref. [7] for the dimensionless nucleus-dependent overlap integrals $V^{(n)}, V^{(p)}, D$ and we denote by $s_{w}=\sin \theta_{w}=0.23$ the weak mixing angle.

\subsection{Minimal field content}

Let us now consider the scenario with minimal field content. By making in Eqs. (29) and (30) the replacement

$$
\frac{v^{4}}{\Lambda_{\mathrm{LFV}}^{4}}\left|\Delta_{i j}\right|^{2} \longrightarrow \frac{\Lambda_{\mathrm{LN}}^{4}}{\Lambda_{\mathrm{LFV}}^{4}}\left|a_{i j}\right|^{2},
$$

one can see that all LFV rates have the following structure

$$
B_{\ell_{i} \rightarrow \ell_{j}(\gamma)}=10^{-50}\left(\frac{\Lambda_{\mathrm{LN}}}{\Lambda_{\mathrm{LFV}}}\right)^{4} R_{\ell_{i} \rightarrow \ell_{j}(\gamma)}\left(s_{13}, \delta ; c^{(i)}\right) .
$$

The overall numerical factor $10^{-50}$ is chosen such that the $R_{\ell_{i} \rightarrow \ell_{j}(\gamma)}$ have a natural size of $\mathcal{O}(1)$. Its value can easily be understood by noting that $\left|a_{i j}\right|^{2} \lesssim\left(\Delta m_{\text {atm }}^{2} / v^{2}\right)^{2} \approx 10^{-52}$.

A glance at the explicit structure of the $a_{i j}$ in Eq. (22) shows that their size is maximized for $s_{13}=s_{13}^{\max }$ (in both normal and inverted hierarchy), due to $\Delta m_{\text {atm }}^{2} \gg$ $\Delta m_{\text {sol }}^{2}$. In order to derive order-of-magnitude conditions on the ratio $\Lambda_{\mathrm{LN}} / \Lambda_{\mathrm{LFV}}$, we consider the reference case defined by $s_{13}=s_{13}^{\max }, \delta=0$, and the reference values quoted 
in Table 1. Then setting all the Wilson coefficients to zero but for $c_{R L}^{(2)}=c_{L L}^{(3)}=1$, and using the overlap integrals and capture rates reported in Ref. [7] (table I of [7]), we find

$B_{\mu \rightarrow e}=\left(\frac{\Lambda_{\mathrm{LN}}}{\Lambda_{\mathrm{LFV}}}\right)^{4}\left\{\begin{array}{ll}6.6 \times 10^{-50} & \text { for } \mathrm{Al} \\ 19.6 \times 10^{-50} & \text { for } \mathrm{Au}\end{array} \quad B_{\mu \rightarrow e \gamma}=8.3 \times 10^{-50}\left(\frac{\Lambda_{\mathrm{LN}}}{\Lambda_{\mathrm{LFV}}}\right)^{4}\right.$.

Despite the strong dependence of the numerical coefficients in Eq. (33) on $s_{13}$, illustrated in Figure 1, these results allow us to draw several interesting conclusions.

- If there is no large hierarchy between the scales of lepton-number and lepton-flavor violation, there is no hope to observe LFV signals in charged-lepton processes. On the other hand, if $\Lambda_{\mathrm{LFV}}$ is not far from the TeV scale (as expected in many realistic scenarios), it is natural to expect visible LFV processes for a wide range of $\Lambda_{\mathrm{LN}}$ : from $10^{13} \mathrm{GeV}$ up to the GUT scale. For instance a $B_{\mu \rightarrow e}=\mathcal{O}\left(10^{-13}\right)$, within reach of the MECO experiment, is naturally obtained for $\Lambda_{\mathrm{LN}} \sim 10^{9} \Lambda_{\mathrm{LFV}}$, which for $\Lambda_{\mathrm{LFV}} \sim 10 \mathrm{TeV}$ implies $\Lambda_{\mathrm{LN}} \sim 10^{13} \mathrm{GeV}$. Such a ratio of scales would also imply $B_{\mu \rightarrow e \gamma}=\mathcal{O}\left(10^{-13}\right)$, within the reach of the MEG experiment.

Note that the requirement of "perturbative" treatment of the couplings $g_{\nu}$, together with upper limits on the light neutrino masses, implies upper limits on the scale $\Lambda_{\mathrm{LN}} \simeq v^{2} g_{\nu} / m_{\nu}$. By loosely requiring $\left|g_{\nu}\right|<1$ one obtains $\Lambda_{\mathrm{LN}} \lesssim$ $3 \times 10^{13}\left(1 \mathrm{eV} / m_{\nu}\right) \mathrm{GeV}$. This means that we cannot make the ratio $\Lambda_{\mathrm{LN}} / \Lambda_{\mathrm{LFV}}$ arbitrarily large.

- Interestingly, $\mu \rightarrow e$ conversion and $\mu \rightarrow e \gamma$ have a quite different sensitivity on the type of operators involved. In particular, while $\mu \rightarrow e \gamma$ is sensitive only to the LR operators, the $\mu \rightarrow e$ conversion is more sensitive to the LL terms:

$$
\begin{aligned}
& \frac{B_{\mu \rightarrow e}\left(c_{R L}^{(2)}=1, \text { other } c^{(i)}=0\right)}{B_{\mu \rightarrow e}\left(c_{L L}^{(3)}=1, \text { other } c^{(i)}=0\right)}= \begin{cases}3 \times 10^{-3} & \text { for } \mathrm{Al}, \\
1.5 \times 10^{-3} & \text { for } \mathrm{Au},\end{cases} \\
& \frac{B_{\mu \rightarrow e}\left(c_{R L}^{(2)}=1, \text { other } c^{(i)}=0\right)}{B_{\mu \rightarrow e}\left(c_{L L}^{(1)}=1, \text { other } c^{(i)}=0\right)}= \begin{cases}0.47 & \text { for } \mathrm{Al}, \\
0.17 & \text { for } \mathrm{Au} .\end{cases}
\end{aligned}
$$

- The comparison of the various $B_{l_{i} \rightarrow l_{j} \gamma}$ rates is a useful tool to illustrate the predictive power of the MLFV (and eventually to rule it out from data). In Figure 2 we report the ratios $B_{\mu \rightarrow e \gamma} / B_{\tau \rightarrow \mu \gamma}$ and $B_{\mu \rightarrow e \gamma} / B_{\tau \rightarrow e \gamma}$ as a function of $s_{13}$ for three different values of the CP violating phase $\delta$ in the normal hierarchy case (the inverted case is obtained by replacing $\delta$ with $\pi-\delta$ ). One observes the clear pattern $B_{\tau \rightarrow \mu \gamma} \gg B_{\tau \rightarrow e \gamma} \sim B_{\mu \rightarrow e \gamma}$, with hierarchy increasing as $s_{13} \rightarrow 0$. Observation of deviations from this pattern could in the future falsify the hypothesis of minimal flavor violation in the lepton sector.

- There is a window in parameter space where we can expect observable effects in $\tau$ decays. As illustrated in Figure 3. $B_{\tau \rightarrow \mu \gamma}$ does not depend on $s_{13}$, while $B_{\mu e}$ does. In the normal hierarchy case, for $\delta=\pi$ and $s_{13} \rightarrow s c \Delta m_{\text {sol }}^{2} / \Delta m_{\text {atm }}^{2}$, one has $B_{\mu \rightarrow e \gamma} \rightarrow 0$. Therefore, close to this region of parameter space one can have a 


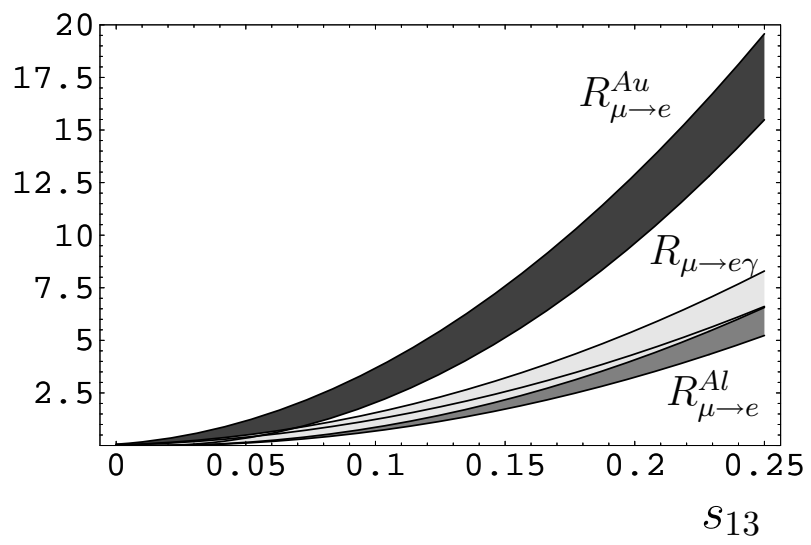

Figure 1: Ratios $R_{i}$ defined in Eq. (32) as a function of $s_{13}$ for $c_{L L}^{(3)}=c_{R L}^{(2)}=1$ and all other $c^{(i)}=0$. The shaded bands correspond to variation of the phase $\delta$ between 0 and $\pi$.
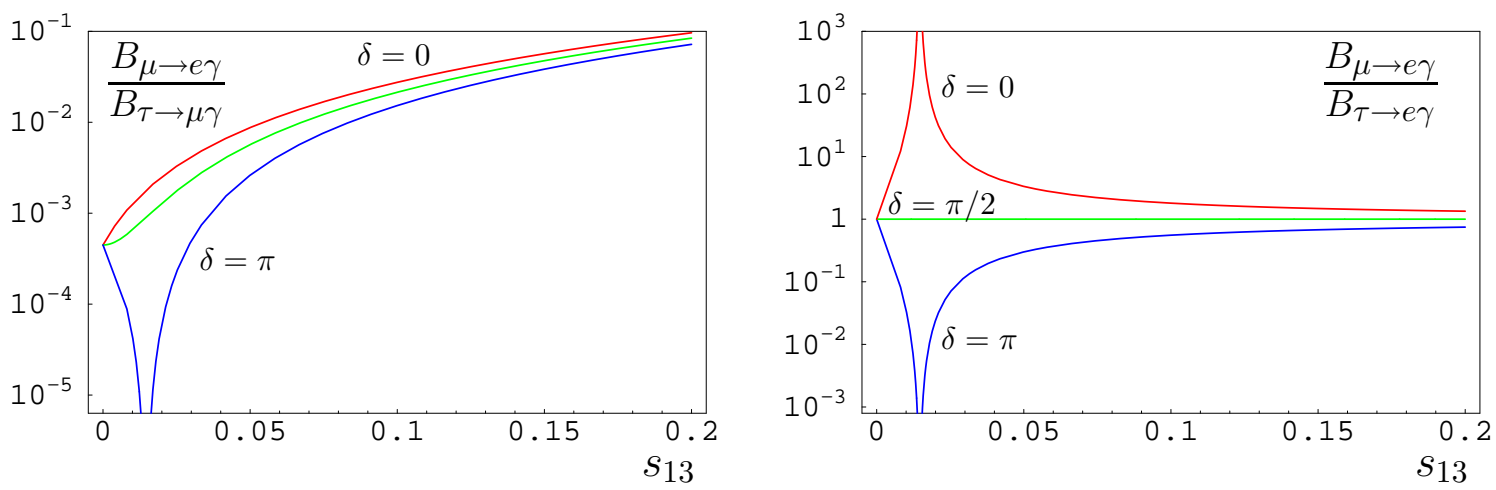

Figure 2: Ratios $B_{\mu \rightarrow e \gamma} / B_{\tau \rightarrow \mu \gamma}$ (left) and $B_{\mu \rightarrow e \gamma} / B_{\tau \rightarrow e \gamma}$ (right) as a function of $s_{13}$ for different values of the $\mathrm{CP}$ violating phase $\delta$ in the normal hierarchy case. The uncertainty due to the first 3 entries in table 1 is not shown.

sizable $B_{\tau \rightarrow \mu \gamma}$ while $B_{\mu \rightarrow e \gamma}$ can be kept below the present experimental limits. In particular, for $\Lambda_{\mathrm{LN}} \sim 10^{10} \Lambda_{\mathrm{LFV}}$ we find $B_{\tau \rightarrow \mu \gamma} \sim 10^{-8}$, which implies a branching ratio for $\tau \rightarrow \mu \gamma$ above $10^{-9}$ possibly observable at (super) $B$ factories. Note that a change in the ratio $\Lambda_{\mathrm{LN}} / \Lambda_{\mathrm{LFV}}$ would only result into a shift of the vertical scale in Figure 3] without affecting the relative distance between the $B_{\mu \rightarrow e \gamma}$ and $B_{\tau \rightarrow \mu \gamma}$ bands.

\subsection{Extended field content}

The discussion of the extended model proceeds in a very similar way, by replacing the dimensionless couplings $a_{i j}$ with the $b_{i j}$, and $\left(\Lambda_{\mathrm{LN}} / \Lambda_{\mathrm{LFV}}\right)^{4}$ with $\left(v M_{\nu} / \Lambda_{\mathrm{LFV}}^{2}\right)^{2}$. The analog of Eq. (32) reads

$$
B_{\ell_{i} \rightarrow \ell_{j}(\gamma)}=10^{-25}\left(\frac{v M_{\nu}}{\Lambda_{\mathrm{LFV}}^{2}}\right)^{2} \widehat{R}_{\ell_{i} \rightarrow \ell_{j}(\gamma)}\left(s_{13}, m_{\nu}^{\text {lightest }} ; c^{(i)}\right) .
$$




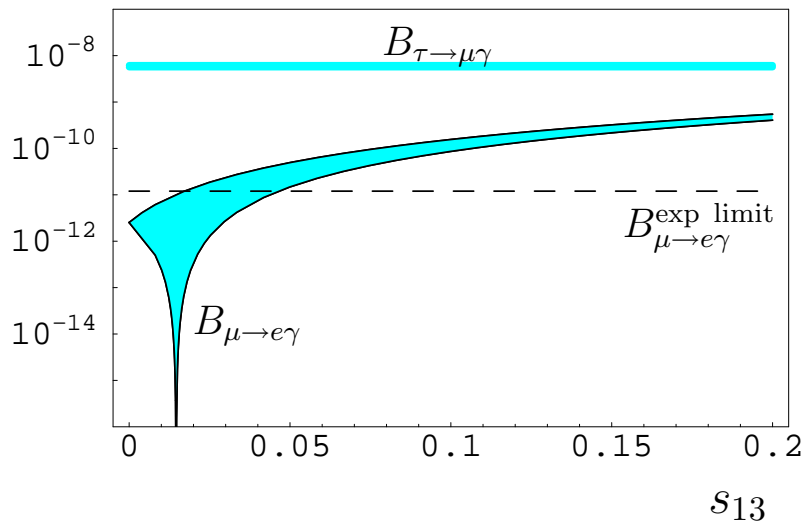

Figure 3: $B_{\tau \rightarrow \mu \gamma}$ and $B_{\mu \rightarrow e \gamma}$ as a function of $s_{13}$, for $\Lambda_{\mathrm{LN}} / \Lambda_{\mathrm{LFV}}=10^{10}$ and $c_{R L}^{(2)}-c_{R L}^{(1)}=1$. The shading corresponds to different values of the phase $\delta$ and the normal/inverted spectrum. The uncertainty due to the first 3 entries in table 1 is not shown.

As illustrated in Figure 4, the dimensionless functions $\widehat{R}_{\ell_{i} \rightarrow \ell_{j}(\gamma)}$ depend on both $s_{13}$ and $m_{\nu_{\text {lightest }}}$, and the maximal values are obtained for $s_{13}=s_{13}^{\max }$ and $m_{\nu_{\text {lightest }}} \rightarrow 0$. In order to explore the sensitivity to the scale ratio $v M_{\nu} / \Lambda_{\mathrm{LFV}}^{2}$, we again pick a favorable reference point $\left(s_{13}=s_{13}^{\max }\right.$ and $\left.m_{\nu_{\text {lightest }}}=0\right)$ and set all the Wilson coefficients to zero except for $c_{R L}^{(2)}=c_{L L}^{(3)}=1$. In the normal hierarchy case we then find

$$
B_{\mu \rightarrow e}=\left(\frac{v M_{\nu}}{\Lambda_{\mathrm{LFV}}^{2}}\right)^{2}\left\{\begin{array}{ll}
1.3 \times 10^{-24} & \text { for } \mathrm{Al} \\
3.7 \times 10^{-24} & \text { for } \mathrm{Au}
\end{array} \quad B_{\mu \rightarrow e \gamma}=1.6 \times 10^{-24}\left(\frac{v M_{\nu}}{\Lambda_{\mathrm{LFV}}^{2}}\right)^{2}\right.
$$

while for the inverted case:

$$
B_{\mu \rightarrow e}=\left(\frac{v M_{\nu}}{\Lambda_{\mathrm{LFV}}^{2}}\right)^{2}\left\{\begin{array}{ll}
6.7 \times 10^{-25} & \text { for } \mathrm{Al} \\
2.0 \times 10^{-24} & \text { for } \mathrm{Au}
\end{array} \quad B_{\mu \rightarrow e \gamma}=8 \times 10^{-25}\left(\frac{v M_{\nu}}{\Lambda_{\mathrm{LFV}}^{2}}\right)^{2}\right.
$$

The general conclusions we can infer from this scenario are the following:

- As was the case with minimal field content, a large hierarchy between $M_{\nu}$ and $\Lambda_{\mathrm{LFV}}$ is required to obtain observable effects. For example, fractions $B_{\mu \rightarrow e(\gamma)}=$ $\mathcal{O}\left(10^{-13}\right)$ are obtained for $M_{\nu} \sim 3 \times 10^{5} \Lambda_{\mathrm{LFV}}^{2} / v$, which for $\Lambda_{\mathrm{LFV}} \sim 10 \mathrm{TeV}$ gives $M_{\nu} \sim 2 \times 10^{11} \mathrm{GeV}$.

- Comparing the values of $M_{\nu}$ in this scenario versus the corresponding values of $\Lambda_{\mathrm{LN}}$ in the minimal case, we find that the same effect in a given LFV process is typically obtained for $\Lambda_{\mathrm{LN}}>M_{\nu}$. This can be understood by noting that in the minimal case the LFV amplitudes are proportional to combinations of the type $\Delta m_{\nu}^{2} \Lambda_{\mathrm{LN}}^{2} / v^{4}$, while in the extended case this factor is replaced by $\Delta m_{\nu} M_{\nu} / v^{2}$. The two scales of lepton number violation are indeed equivalent when this factor is of $\mathcal{O}(1)$, namely for the maximal value allowed by the "perturbative condition" on the Yukawa couplings: $M_{\nu} \sim \Lambda_{\mathrm{LN}} \sim v^{2} / \Delta m_{\nu} \sim 10^{15} \mathrm{GeV}$. 

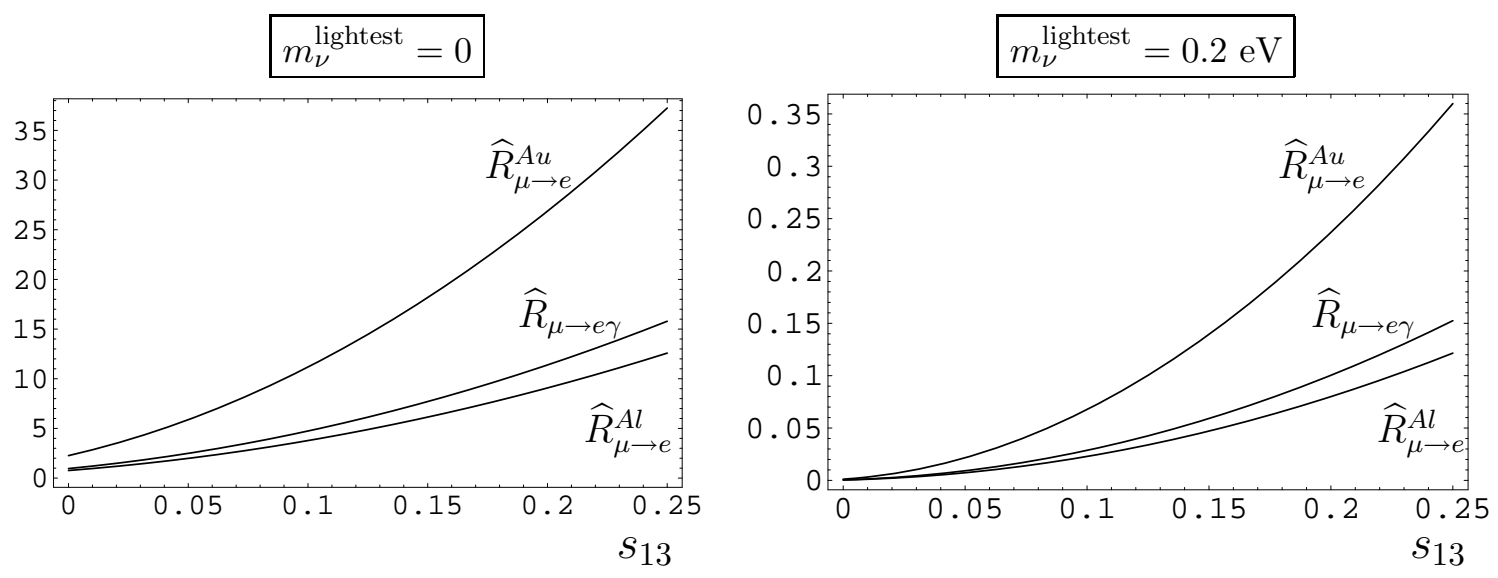

Figure 4: Ratios $\widehat{R}_{i}$ defined in Eq. (36) as a function of $s_{13}$ for $c_{L L}^{(3)}=c_{R L}^{(2)}=1$, all other $c^{(i)}=0$, normal spectrum and $\delta=0$.
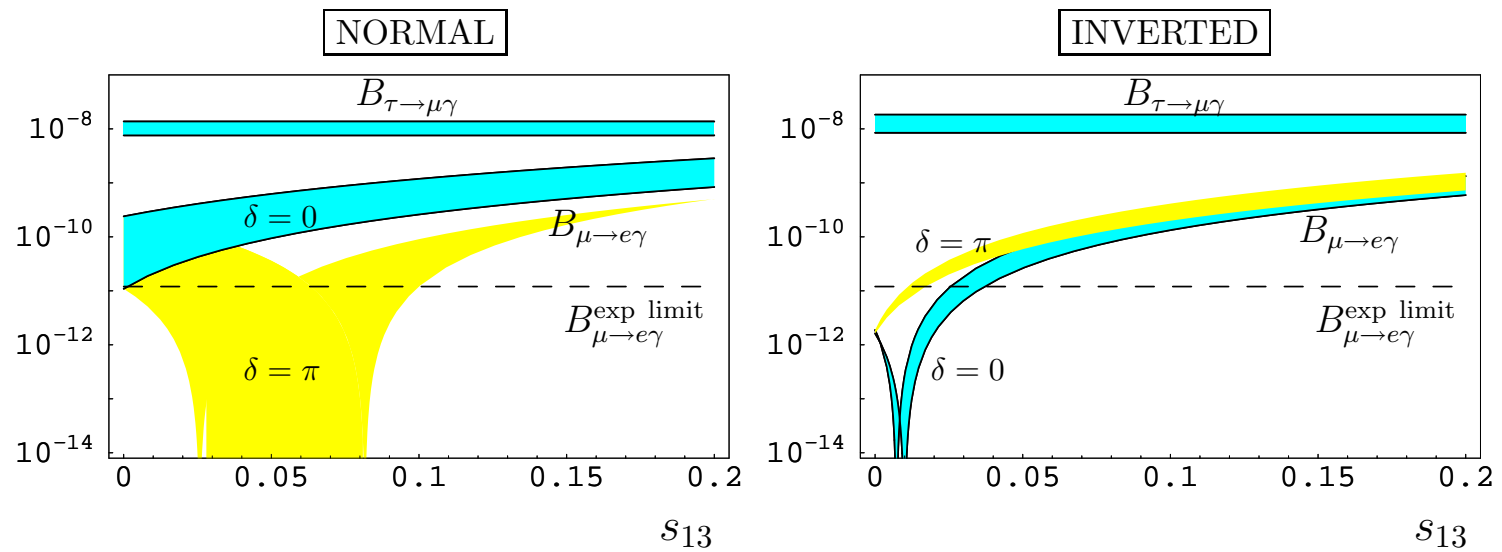

Figure 5: $\quad B_{\tau \rightarrow \mu \gamma}$ and $B_{\mu \rightarrow e \gamma}$ as a function of $s_{13}$, for $\left(v M_{\nu}\right) / \Lambda_{\mathrm{LFV}}^{2}=5 \times 10^{7}$ and $c_{R L}^{(2)}-c_{R L}^{(1)}=1$. The shading corresponds to different values of the lightest neutrino mass, ranging between 0 and $0.02 \mathrm{eV}$. The two choices of $\delta$ correspond to the \pm sign in Eq. (24).

- Much like in the previous scenario, in this case the ratios of the various LFV rates are unambiguously determined in terms of neutrino masses and mixing angles; however, the results are potentially different than in the minimal case because of the different relation between LFV parameters and neutrino mass matrix. As illustrated in Fig. [5, one still observes the pattern $B_{\tau \rightarrow \mu \gamma} \gg B_{\mu \rightarrow e \gamma}\left(\sim B_{\tau \rightarrow e \gamma}\right)$. For a given choice of $\delta=0$ or $\pi$, the strength of the $\mu \rightarrow e$ suppression is very sensitive to whether the hierarchy is normal or inverted. For $\delta=0$ the present experimental limit on $B_{\mu \rightarrow e \gamma}$ allows large values of $B_{\tau \rightarrow \mu \gamma}$ only for the inverted hierarchy, whereas for $\delta=\pi$, a large region with a sizable $B_{\tau \rightarrow \mu \gamma}$ is allowed only for the normal hierarchy. Note that the overall vertical scale of Fig. 5 depends on both the ratio $\left(v M_{\nu}\right) / \Lambda_{\mathrm{LFV}}^{2}$ - as implied by Eq. 36] - and the value of the lightest neutrino mass (as illustrated by Fig. 4). 


\section{Conclusions}

In this paper we extend the notion of MFV to the lepton sector. We define a symmetry principle and an effective field theory which allows us to relate, in a general way, leptonflavor mixing in the neutrino sector to lepton-flavor violation in the charged lepton sector. The construction of such effective theory allow us to address fundamental questions about the flavor structure of the lepton sector in a very general way, insensitive to many details about the physics beyond the SM.

We find two ways in which we can define the sources of flavor symmetry breaking in the lepton sector in a minimal and thus very predictive way: i) a scenario where the lefthanded Majorana mass matrix is the only irreducible source of flavor symmetry breaking; and ii) a scenario with heavy right-handed neutrinos, where the Yukawa couplings define the irreducible sources of flavor symmetry breaking and the right-handed Majorana mass matrix has a trivial flavor structure.

We find that visible LFV effects in the charged lepton sector are generated when there is a large hierarchy between the scales of lepton flavor mixing $\left(\Lambda_{\mathrm{LFV}}\right)$ and total lepton number violation $\left(\Lambda_{\mathrm{LN}}\right)$. This condition is indeed realized within the explicit extensions of the SM widely discussed in the literature which predict sizable LFV effects in charged leptons [4, 10] (for an updated discussion see Ref. 11] and references therein). Within our general framework, we find that the new generation of experiments on LFV would naturally probe the existence of new degrees of freedom carrying lepton flavor numbers up to energy scales of the order of $10^{3} \mathrm{TeV}$, if the scale $\Lambda_{\mathrm{LN}}$ is close to the GUT scale.

The two scenarios we have considered are highly predictive and possibly testable by future experiments. While the rates for LFV effects strongly depend on the (unknown) scales $\Lambda_{\mathrm{LN}}$ and $\Lambda_{\mathrm{LFV}}$, the ratio of different LFV rates are unambiguously predicted in terms of neutrino masses and mixing angles. At present, the uncertainty in our predictions for such ratios arises mainly from the poorly constrained value of $s_{13}$ and, to a lesser extent, from the neutrino spectrum ordering and the CP violating phase $\delta$.

One of the clearest consequences from the phenomenological point of view is that if $s_{13} \gtrsim 0.1$ there is no hope to observe $\tau \rightarrow \mu \gamma$ at future accelerators. On the other hand, $\mu \rightarrow e \gamma$ and $\mu$-to-e conversion are within reach of future experiments for reasonable values of the symmetry breaking scales.

Acknowledgments We thank Curtis Callan for valuable discussions. V.C. was supported by Caltech through the Sherman Fairchild fund, and acknowledges the hospitality of the LNF Spring Institute 2005. This work was supported by the U.S. Deartment of Energy under grants DE-FG03-97ER40546 and DE-FG03-92ER40701. 


\section{References}

[1] R. S. Chivukula and H. Georgi, Phys. Lett. B 188, 99 (1987).

[2] A. J. Buras et al., Phys. Lett. B 500, 161 (2001) hep-ph/0007085.

[3] G. D’Ambrosio, G. F. Giudice, G. Isidori and A. Strumia, Nucl. Phys. B 645, 155 (2002) hep-ph/0207036.

[4] F. Borzumati and A. Masiero, Phys. Rev. Lett. 57, 961 (1986);

J. Hisano, T. Moroi, K. Tobe and M. Yamaguchi, Phys. Rev. D 53, 2442 (1996) hep-ph/9510309.

[5] S. Yamada, Nucl. Phys. Proc. Suppl. 144, 185 (2005) http://meg.web.psi.ch/

[6] J. L. Popp [MECO Collaboration], AIP Conf. Proc. 721, 321 (2004) http://meco.ps.uci.edu/

[7] R. Kitano, M. Koike and Y. Okada, Phys. Rev. D 66, 096002 (2002) hep-ph/0203110.

[8] A. Strumia and F. Vissani, hep-ph/0503246.

[9] S. Eidelman et al. [Particle Data Group], Phys. Lett. B 592, 1 (2004).

[10] R. Barbieri, L. J. Hall and A. Strumia, Nucl. Phys. B 445, 219 (1995) hep-ph/9501334.

[11] A. Masiero, S. K. Vempati and O. Vives, New J. Phys. 6, 202 (2004) hep-ph/0407325. 


\section{Appendix}

\begin{tabular}{|c|c|c|c|c|}
\hline & $V^{(p)}$ & $V^{(n)}$ & $D$ & $\Gamma_{\text {capt }}\left(10^{6} \mathrm{~s}^{-1}\right)$ \\
\hline${ }_{13}^{27} \mathrm{Al}$ & 0.0161 & 0.0173 & 0.0362 & 0.7054 \\
\hline${ }_{79}^{197} \mathrm{Au}$ & 0.0974 & 0.146 & 0.189 & 13.07 \\
\hline
\end{tabular}

Table 2: Reference values of nuclear overlap integrals and capture rates used in the phenomenological analysis [7]. The overlap integrals are those computed using "Method I" in Ref. [7].

We use the results of Kitano et al. [7] on the rate of $\mu$-to-e conversion in various nuclei for the numerical results in this paper. In this appendix we summarize the results from Ref. [7] that we use in this paper. In terms of effective couplings $\tilde{g}_{L V}^{(p, n)}, A_{R}$, and of nuclear overlap integrals $D, V^{(p, n)}$, the branching ratio for the radiative decay is

$$
B_{\mu \rightarrow e \gamma}=384 \pi^{2}\left|A_{R}\right|^{2}
$$

and the branching ratio for conversion in nuclei is

$$
B_{\mu \rightarrow e}=\frac{2 G_{F}^{2} m_{\mu}^{5}}{\Gamma_{\text {capt }}}\left|A_{R}^{*} D+\tilde{g}_{L V}^{(p)} V^{(p)}+\tilde{g}_{L V}^{(n)} V^{(n)}\right|^{2}
$$

The numerical values of the nuclear overlap integrals used in this paper are given in Table 2.

The relations between the effective couplings $\tilde{g}_{L V}^{(p, n)}, A_{R}$, and the coefficients in the effective Lagrangian, Eq. (28), are:

$$
\begin{aligned}
\tilde{g}_{L V}^{(p)} & =-\frac{4 v^{2}}{\Lambda_{\mathrm{LFV}}^{2}} \Delta_{\mu e}^{*}\left[\left(\frac{1}{4}-s_{w}^{2}\right)\left(c_{L L}^{(1)}+c_{L L}^{(2)}\right)+\frac{3}{2} c_{L L}^{(3)}+c_{L L}^{(4 u)}+\frac{1}{2} c_{L L}^{(4 d)}-\frac{1}{2} c_{L L}^{(5)}\right] \\
\tilde{g}_{L V}^{(n)} & =-\frac{4 v^{2}}{\Lambda_{\mathrm{LFV}}^{2}} \Delta_{\mu e}^{*}\left[-\frac{1}{4}\left(c_{L L}^{(1)}+c_{L L}^{(2)}\right)+\frac{3}{2} c_{L L}^{(3)}+\frac{1}{2} c_{L L}^{(4 u)}+c_{L L}^{(4 d)}+\frac{1}{2} c_{L L}^{(5)}\right] \\
A_{R} & =\frac{e v^{2}}{\Lambda_{\mathrm{LFV}}^{2}} \Delta_{\mu e}\left[-c_{R L}^{(1)}+c_{R L}^{(2)}\right]
\end{aligned}
$$

